\title{
A Modified Method for Modeling of Heterogeneous Hydrodynamics Processes
}

https://doi.org/10.31713/MCIT.2020.06

\author{
Gennadiy Sandrakov \\ Faculty of Computer Science and Cybernetics \\ Taras Shevchenko National University of Kyiv \\ Kyiv, Ukraine \\ gsandrako@gmail.com
}

\begin{abstract}
A modified method of numerical modeling for heterogeneous fluid dynamics processes with take of phase transitions will be presented. The method is based on the homogenization on cells and approximation of conservation laws for masses, momentums, and energies in integral and differential forms. The combination of Harlow's particle-in-cell method, Belotserkovskii's large particles method and Bakhvalov's homogenization method is used for computing by the modified method simulation for processes with phase transitions.
\end{abstract}

Keywords-modeling methods, hydrodynamics processes, phase transitions, conservation laws, homogenization methods.

\section{INTRODUCTION}

A modified numerical method of simulations for processes of heterogeneous hydrodynamics with take of phase transitions will be discussed. It is supposed that the fluids are compressible and inviscid (non-viscous). Heterogeneities of the fluids are considered as small drops or particles of one fluid within other fluid. Total number of the drops may be large enough and the drops may have phase transitions. Thus simulations of the main fluid with small transited drops dynamics are considered. These are dynamics of multiphase flows really. Therefore it is possible to use general multiphase flow models in the case. However, relevant equations are not complete as a rule. For example, there is a problem as to distribute energies between the phases in the model dynamics. Various physical experiments are necessary for solving of the problem in concrete cases. The situation is more difficult whenever phase transitions are admissible.

The modified numerical simulation method is based on homogenization on cells and relevant approximation of conservation laws for masses, momentums, and energies in integral and differential forms. The approximation is natural and numerical simulations are realized as direct computer experiments. The method seems to be much more adequate to the physical and mathematical essence of the dynamics because conservation laws are correct on the discrete level.

The relevance of this problem is due to the intensive development of the branches of physics, chemistry and mechanics related to the study of dynamic processes occurring under pulsed loading and the passage of strong shock waves in metals, polymers, composites and other solids. The study and optimization of such processes are necessary for the development of new technologies for the synthesis of new substances. The formation of new substances, their modifications and phases is associated with physicochemical processes initiated at high pressures and energies. The calculation of such wave processes is complicated, since these physicochemical processes strongly affect the behavior of the initiating waves. In addition, phase transitions under the action of waves loading can lead to multi-front waves. To analyze these processes, the development of mathematical models are necessary, taking into account the basic principles of the theory of phase transformations, and the construction of highly efficient numerical methods and computational algorithms..

\section{The PARTICLE MethodS}

The modified method is a combination of the Harlow's particle-in-cell method, Belotserkovskii's large particles method and Bakhvalov's homogenization method (see [1] and [2]), where Euler's and Lagrange's approaches are used simultaneously. Some backgrounds of the combination were presented in [3-6] and developed in [7] partially. Let us recall some background of the methods before to give more details on the method combination. Euler's and Lagrange's approaches are used simultaneously in the particle-in-cell method for homogeneous fluid (or gas). The method is based on an approximation of conservation laws for masses, momentums, and energies of the fluid in the following integral forms

$$
\begin{aligned}
& \cdot \int_{V(t)} \rho_{t}^{\prime} d \tau=-\int_{S(t)}(\rho W) \cdot N d s, \\
& \frac{d}{d t} \int_{V(t)} \rho W d \tau=-\int_{S(t)} p N d s, \\
& \frac{d}{d t} \int_{V(t)} \rho E d \tau=-\int_{S(t)}(p W) \cdot N d s,
\end{aligned}
$$

where $V(t)$ and $S(t)$ are volume and surface of some Lagrange's domain in the fluid, $N$ is an exterior normal to the domain, $p=p(\rho, E)$ and $\rho, W, E$ are unknown density, velocity, and full energy. For example, the case of three 


\section{Modeling, control and information technologies -2020}

dimension space may be discussed and therefore by definition ones have $W=(u, v, w)$ for the velocity vector function.

It is known [1] that the conservation laws are equivalent to conservation laws for masses, momentums, and energies of the fluid in the following differential forms

$$
\begin{gathered}
\frac{\partial \rho}{\partial t}+\operatorname{div}(\rho W)=0, \\
\frac{\partial \rho W}{\partial t}+\operatorname{div}(\rho W \otimes W)+\nabla p=0, \\
\frac{\partial \rho E}{\partial t}+\operatorname{div}(\rho E W)+\operatorname{div}(p W)=0,
\end{gathered}
$$

where $W \otimes W$ is the tensor square of vector function $W=W(t, x, y, z)$ and $x, y, z$ is a point of some domain $\Omega$, which is filled by the heterogeneous fluid under consideration.

The time approximation in the particle-in-cell method is natural. Simulations are conducted step by step with a small enough time interval $\Delta t$ that starting from an initial configuration. The space approximation in the method is more complicated and dynamics are taking into consideration. The fluid region $\Omega$ is divided into cells with the small size $\Delta x$ and the fluid filling every such cell is considered as a collection of a few particles or drops. Every such particle have own mass, volume, energy, and coordinates that are specified at an initial moment. In addition the density, velocity, and full energy $\rho^{n}, u^{n}, v^{n}, w^{n}, E^{n}$ are specified for every such cell with number $i, j, k$ at the time moment $t=n \Delta t$.

Corresponding time step of the simulation is split up to three stages so the discrete conservation laws are faithful. For example, the total mass of particles under consideration is saved at every time step of such approximation.

On the first stage of the time step, the auxiliary velocity $\vec{u}^{n}, \bar{v}^{n}, \bar{w}^{n}$ and the energy $\bar{E}^{n}$ of particles from the sell with number $i, j, k$ are calculated by the following formulas

$$
\begin{gathered}
\bar{u}_{i, j, k}^{n}=u_{i, j, k}^{n}-\frac{p_{i+1, j, k}^{n}-p_{i-l, j, k}^{n}}{2 \Delta x} \frac{\Delta t}{\rho_{i, j, k}^{n}}, \\
v_{i, j, k}^{n}=v_{i, j, k}^{n}-\frac{p_{i, j+1, k}^{n}-p_{i, j-1, k}^{n}}{2 \Delta y} \frac{\Delta t}{\rho_{i, j, k}^{n}}, \\
\bar{w}_{i, j, k}^{n}=w_{i, j, k}^{n}-\frac{p_{i, j, k+1}^{n}-p_{i, j, k-1}^{n}}{2 \Delta z} \frac{\Delta t}{\rho_{i, j, k}^{n}}, \\
\bar{E}_{i, j, k}^{n}=E_{i, j, k}^{n}- \\
-\frac{p_{i+1 / 2, j, k}^{n} u_{i+1 / 2, j, k}^{n}-p_{i-1 / 2, j, k}^{n} u_{i-1 / 2, j, k}^{n}}{\Delta x} \frac{\Delta t}{\rho_{i, j, k}^{n}}-
\end{gathered}
$$

$$
\begin{gathered}
-\frac{p_{i, j+1 / 2, k}^{n} u_{i, j+1 / 2 . k}^{n}-p_{i, j-1 / 2, k}^{n} u_{i, j-1 / 2, k}^{n}}{\Delta y} \frac{\Delta t}{\rho_{i, j, k}^{n}}- \\
-\frac{p_{i, j, k+1 / 2}^{n} u_{i, j, k+1 / 2}^{n}-p_{i, j, k-1 / 2}^{n} u_{i, j, k-1 / 2}^{n}}{\Delta z} \frac{\Delta t}{\rho_{i, j, k}^{n}},
\end{gathered}
$$

where

$$
\begin{aligned}
& p_{i+1 / 2, j, k}^{n}=\frac{p_{i+1, j, k}^{n}-p_{i, j, k}^{n}}{2}, p_{i-1 / 2, j, k}^{n}=\frac{p_{i, j, k}^{n}-p_{i-1, j, k}^{n}}{2}, \\
& u_{i+1 / 2, j, k}^{n}=\frac{u_{i+1, j, k}^{n}-u_{i, j, k}^{n}}{2}, u_{i-1 / 2, j, k}^{n}=\frac{u_{i, j, k}^{n}-u_{i-1, j, k}^{n}}{2}, \\
& v_{i, j+1 / 2, k}^{n}=\frac{v_{i, j+1, k}^{n}-v_{i, j, k}^{n}}{2}, v_{i, j-1 / 2, k}^{n}=\frac{v_{i, j, k}^{n}-v_{i, j-1, k}^{n}}{2}
\end{aligned}
$$

and similar formula is used for $p_{i, j+1 / 2, k}^{n}, \ldots, w_{i, j, k-1 / 2}^{n}$.

This is the Euler's stage for approximations of transport free momentums equations in (1) by the pressure forces for every sell. On the second stage, motions of the particles by the velocities are taking into account. This is the Lagrange's stage for an approximation of masses equation that is modeling of mass transports from a sell to surrounding sells. On the third stage, moving of the momentums and energy are calculated. This is the concluding stage for approximations of pressure free momentums and energies equations in (1) that are modeling of the momentums and energy transports by the dynamics from a sell to surrounding sells.

The approximations are rationale from physical and mathematical point of view since conservation laws are correct on the discrete levels during the courses of corresponding numerical simulations. Therefore the particle-in-cells method is effective enough for numerical evaluations of homogeneous fluid (or gas) dynamics by boundary conditions and external forces. Concrete types of fluid are defined here by a form of state equation $p=p(\rho, J)$, where $J=E-W^{2} / 2$ is a notation for interior energy of homogeneous fluid (or gas).

An essential problem of the method is only that total number of particles may be very large. Indeed total number of cells must be large enough for best approximations and the number of particles at every cell must be large enough also. Moreover every such particle must have own mass, volume, energy, and coordinates. Thus there is massive data and the data is recalculated from step to step in the simulation.

In order to avoid the problem it is possibly to use the large particles method. The time approximation in the method is similar to the approximation in the particle-in-cell method. The space approximation in the method is following. A fluid region is divided into cells with small size and the fluid filling every such cell is considered as a large particle or drop. Every such particle have own mass, volume and energy that are specified at an initial moment. In addition the density, velocity, and full energy are specified for every such cell at the moment. But the volume of particle is coincided with the volume of cell now, 


\section{Modeling, control and information technologies -2020}

where homogenization is used. Therefore the mass and energy of particle are defined by the density and full energy. Thus the data is not so massive in the method by the homogenization.

Corresponding time step of the simulation is split up to three stages also and so approximate conservation laws are faithful. The stages are similar to the stages of the particles in cells method, for example, formulas (3) are used on the first stage. Modifications are need only for modeling of the mass and momentums transports by the dynamics. For example, the mass transports are calculated as moving of corresponding share of large particle mass from the cell to corresponding surrounding sell by the following formulas

$$
\begin{aligned}
M_{i+1 / 2, j, k}^{n} & =\bar{\rho}_{i+1 / 2, j, k}^{n} \bar{u}_{i+1 / 2, j, k}^{n} \Delta y \Delta z \Delta t, \\
M_{i-1 / 2, j, k}^{n} & =\bar{\rho}_{i-1 / 2, j, k}^{n} \bar{u}_{i-1 / 2, j, k}^{n} \Delta y \Delta z \Delta t, \\
M_{i, j+1 / 2, k}^{n} & =\bar{\rho}_{i, j+1 / 2, k}^{n} v_{i, j+1 / 2, k}^{n} \Delta x \Delta z \Delta t, \\
M_{i, j-1 / 2, k}^{n} & =\bar{\rho}_{i, j-1 / 2, k}^{n} v_{i, j-1 / 2, k}^{n} \Delta x \Delta z \Delta t, \\
M_{i, j, k+1 / 2}^{n} & =\bar{\rho}_{i, j, k+1 / 2}^{n} \bar{w}_{i, j, k+1 / 2}^{n} \Delta x \Delta y \Delta t, \\
M_{i, j, k-1 / 2}^{n} & =\bar{\rho}_{i, j, k-1 / 2}^{n} \bar{w}_{i, j, k-1 / 2}^{n} \Delta x \Delta y \Delta t,
\end{aligned}
$$

where $\bar{u}_{i+1 / 2, j, k}^{n}, \bar{u}_{i-1 / 2, j, k}^{n}, \bar{v}_{i, j+1 / 2, k}^{n}, \ldots, \bar{w}_{i, j, k-1 / 2}^{n}$ are calculated as in (3) and we use the equalities

$$
\begin{aligned}
& \bar{\rho}_{i+1 / 2, j, k}^{n}= \begin{cases}\rho_{i, j, k}^{n}, & \text { if } \bar{u}_{i+1 / 2, j, k} \geq 0, \\
\rho_{i+1, j, k}^{n}, & \text { if } u_{i+1 / 2, j, k}^{n}<0,\end{cases} \\
& \bar{\rho}_{i-1 / 2, j, k}^{n}=\left\{\begin{array}{c}
\rho_{i-1, j, k}^{n}, \text { if } \bar{u}_{i-1 / 2, j, k} \geq 0, \\
\rho_{i, j, k}^{n}, \text { if } u_{i-1 / 2, j, k}^{n}<0,
\end{array}\right.
\end{aligned}
$$

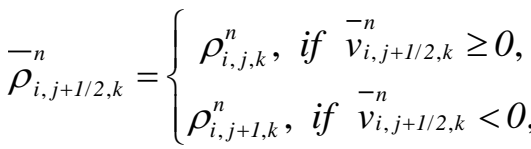

$$
\begin{aligned}
& \bar{\rho}_{i, j-1 / 2, k}^{n}= \begin{cases}\rho_{i, j-1, k}^{n}, & \text { if } \bar{v}_{i, j-1 / 2, k} \geq 0, \\
\rho_{i, j, k}^{n}, & \text { if } v_{i, j-1 / 2, k}<0,\end{cases} \\
& \bar{\rho}_{i, j, k+1 / 2}^{n}=\left\{\begin{array}{l}
\rho_{i, j, k}^{n}, \text { if } \bar{w}_{i, j, k+1 / 2}^{n} \geq 0, \\
\rho_{i, j, k+1}^{n}, \text { if } \bar{w}_{i, j, k+1 / 2}^{n}<0,
\end{array}\right. \\
& \bar{\rho}_{i, j, k-1 / 2}^{n}=\left\{\begin{array}{c}
\rho_{i, j, k-1}^{n}, \text { if } \bar{w}_{i, j, k-1 / 2}^{n} \geq 0, \\
\rho_{i, j, k}^{n}, \text { if } \bar{w}_{i, j, k-1 / 2}^{n}<0 .
\end{array}\right.
\end{aligned}
$$

The formulas (4) and (5) are actually an approximation for the remaining equations from (2), which express the conservation laws of momentum and energy, under the assumption that the terms with divergence are equal to zero. Accordingly, the approximation of such terms with divergence determining the momentum transfer and energy is produced at the final stage in the transition from the time step to a new step.
On the third stage, moving of the momentums $u^{n+1}, v^{n+1}, w^{n+1}$ and the energy $E^{n+1}$ on the time step $n+1$ are calculated by the following formulas

$$
\begin{aligned}
& \rho_{i, j, k}^{n+1}=\rho_{i, j, k}^{n}- \\
& -\mathrm{O}_{x y z}^{-1}\left\{M_{i+1 / 2, j, k}^{n}-M_{i-1 / 2, j, k}^{n}+M_{i, j+1 / 2, k}^{n}-\right. \\
& \left.-M_{i, j-1 / 2, k}^{n}+M_{i, j, k+1 / 2}^{n}-M_{i, j, k-1 / 2}^{n}\right\}, \\
& u_{i, j, k}^{n+1}=\frac{\rho_{i, j, k}^{n}}{\rho_{i, j, k}^{n+1}} u_{i, j, k}^{n}- \\
& -\mathrm{O}_{x y z}^{-1}\left\{\stackrel{-n}{u_{i+1 / 2, j, k}} M_{i+1 / 2, j, k}^{n}-{ }^{n} u_{i-1 / 2, j, k} M_{i-1 / 2, j, k}^{n}+\right. \\
& \begin{array}{l}
-n \\
+u_{i, j+1 / 2, k}
\end{array} M_{i, j+1 / 2, k}^{n}-\bar{u}_{i, j-1 / 2, k}^{n} M_{i, j-1 / 2, k}^{n}+ \\
& \left.\begin{array}{l}
-n \\
+u_{i, j, k+1 / 2} M_{i, j, k+1 / 2}^{n}
\end{array}-\bar{u}_{i, j, k-1 / 2}^{n} M_{i, j, k-1 / 2}^{n}\right\}, \\
& v_{i, j, k}^{n+1}=\frac{\rho_{i, j, k}^{n}}{\rho_{i, j, k}^{n+1}} v_{i, j, k}^{n}- \\
& -\mathrm{O}_{x y z}^{-1}\left\{\begin{array}{l}
-n \\
v_{i+1 / 2, j, k}
\end{array} M_{i+1 / 2, j, k}^{n}-{\stackrel{-n}{v_{i-1 / 2, j, k}}}^{n} M_{i-1 / 2, j, k}^{n}+\right.
\end{aligned}
$$

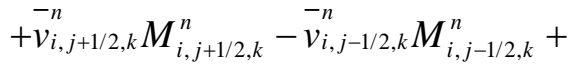

$$
\begin{aligned}
& \left.\begin{array}{rl} 
& -n \\
+v_{i, j, k+1 / 2} & M_{i, j, k+1 / 2}^{n}-v_{i, j, k-1 / 2}^{n} M_{i, j, k-1 / 2}^{n}
\end{array}\right\}, \\
& w_{i, j, k}^{n+1}=\frac{\rho_{i, j, k}^{n}}{\rho_{i, j, k}^{n+1}} w_{i, j, k}^{n}- \\
& -\mathrm{O}_{x y z}^{-1}\left\{\begin{array}{l}
-n \\
w_{i+1 / 2, j, k}
\end{array} M_{i+1 / 2, j, k}^{n}-\bar{w}_{i-1 / 2, j, k}^{n} M_{i-1 / 2, j, k}^{n}+\right.
\end{aligned}
$$

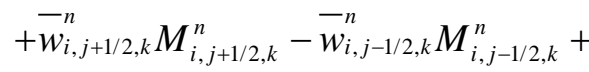

$$
\begin{aligned}
& \left.\begin{array}{l}
{ }^{n} \\
+w_{i, j, k+1 / 2} \\
M_{i, j, k+1 / 2}^{n}
\end{array}-\bar{w}_{i, j, k-1 / 2}^{n} M_{i, j, k-1 / 2}^{n}\right\}, \\
& E_{i, j, k}^{n+1}=\frac{\rho_{i, j, k}^{n}}{\rho_{i, j, k}^{n+1}} E_{i, j, k}^{n}- \\
& -\mathrm{O}_{x y z}^{-1}\left\{\bar{E}_{i+1 / 2, j, k}^{n} M_{i+1 / 2, j, k}^{n}-\bar{E}_{i-1 / 2, j, k}^{n} M_{i-1 / 2, j, k}^{n}+\right. \\
& +\bar{E}_{i, j+1 / 2, k}^{n} M_{i, j+1 / 2, k}^{n}-\bar{E}_{i, j-1 / 2, k}^{n} M_{i, j-1 / 2, k}^{n}+ \\
& \left.+\bar{E}_{i, j, k+1 / 2}^{n} M_{i, j, k+1 / 2}^{n}-\bar{E}_{i, j, k-1 / 2}^{n} M_{i, j, k-1 / 2}^{n}\right\},
\end{aligned}
$$




\section{Modeling, control and information technologies -2020}

The modified numerical method of simulations is designed to numerical modeling of the main fluid with small transited drops dynamics. The time approximation in the method is as in above methods. The space approximation in the method is following. Heterogeneous fluid region is divided into cells with small size. The main fluid filling every such cell is considered as a large particle while the transited drops are considered as the collection of a few "small" particles in the cell. Every large particle have own mass, volume and energy that are specified at an initial moment by the homogenization. Every small particle have own mass, volume, energy, and coordinates that are specified at the moment. In addition the density, velocity, and full energy are specified for every such cell. This is a combination of above methods at the initial moment. Corresponding time step of the simulation is split up to three stages as described above with additional averaging stage.

On the averaging stage, energies of large particle and small particles in every cell are distributed between the particles so that a pressure in the cell is uniform. Indeed the large particle induce some pressure by own state equation and the small particles induce some pressure by own state equation and it is natural to distribute energies of the particles so that the first pressure coincides with second pressure. Moreover on the stage, it is possible to observe phase transitions of the small particles by the pressure, for example. The phase transitions are realized if the pressure is more than critical pressure by the corresponding phase diagram. In the case the small particles may change own volume, energy, and state equation. Thus the heterogeneous fluid may have three or more phases. Thus, we use formulas (3) on the stage to calculate the intermediate velocities $\bar{u}^{n}, v, \bar{w}^{n}$ and the energy $\bar{E}^{n}$ of particles from the sell with number $i, j, k$ at the time moment $t=n \Delta t$. The remaining stages are similar to the stages of above methods. For example, the mass transports are calculated as moving of corresponding share of large particle mass and small particles masses from the cell to corresponding surrounding sell by formulas (5), which are used in (6) by homogenization.

Therefore total mass of the heterogeneous fluid under consideration is saved at every time step of such numerical approximation. The momentums $u^{n+1}, v^{n+1}, w^{n+1}$ and the energy $E^{n+1}$ on the step $n+1$ are modeling in according formulas (6) by homogenization. Thus, this is a combination of above methods from step to step during the courses of corresponding numerical simulations. The method seems to be reasonable for numerical evaluations of such heterogeneous fluid (or gas) dynamics and the recalculated data is not very massive. On the other hand it is possible to use the particles in cells method with the preliminary stages for modeling of the dynamics. But the recalculated data is very massive in the case.

The relations complete the description of the modified numerical method of simulations for calculating all parameter values, which describe the dynamics of the heterogeneous fluid under consideration with inclusions on a new time step. Further calculations are conducted step by step with a small enough time interval $\Delta t$ that starting from an initial configuration.

\section{NUMERICAL MODELING}

The presented modified method is designed to numerical modeling of following physical processes. Let consider graphite drops distributing uniformly in some fluid. More exactly, there is heterogeneous medium with graphite particles and the medium may be considered under high pressure as "fluid" with corresponding state equation. For example, we consider a cylinder of the medium that consist of copper with graphite particles. Let the cylinder be in an outside explosive tube device. Inducing detonation shock waves in the outside explosive tube device, we can observe dynamics of such shock waves in computer experiments by the method.

Some computer experiment results may be found in [3]. The results were in agreement with known results of physical experiments. More details of the presented modified method and other modifications may be found in papers [4,5,7].

The modified method was also applied to numerical simulations of plasma dynamics according to [6]. The plasma may be considered as gas with ionized particles. The gas and particles were defined by corresponding state equations. Equations (2) were coupled with Maxwell's equations and on the discrete level also. Inducing motions of the heterogeneous plasma in some region it was possible to observe absorption of the ionized particles on relevant boundaries in computer experiments by the method coupling with appropriate method for Maxwell's equations [1,6]. Alternative methods and corresponding references for the problem may be found in [8].

Moreover, the presented modified method seems to be perspective for numerical simulations of other absorption and diffusion processes in complex fluid and plasma dynamics.

\section{REFERENCES}

[1] O. M. Belotserkovskij, Yu. M. Davidov, The Large Particles Method in Gas Dynamics. Nauka, Moscow, 1982. (in Russian).

[2] N.S. Bakhvalov, G. Panasenko, Homogenisation: Averaging Processes in Periodic Media. Kluwer Academic Publishers, Springer Netherlands, 1989.

[3] S. V. Boyko, G. V. Sandrakov, "Parameter computing of hydrodynamics processes with phase transitions," Bulletin of Taras Shevchenko National University of Kyiv, Series: Physics \& Mathematics, Special no. , pp. 11-16, 2013.

[4] G. V. Sandrako, S. B. Boyko, "Mathematical modeling of complex heterogeneous fluid dynamics," Journal Num. and Appl. Math., № 1 (104), pp. 109-120, 2011. (in Russian).

[5] S. B. Boyko, G. V. Sandrako, "Parameter computing of hydrodynamics processes with phase transitions," Journal Num. and Appl. Math., № 2 (108), pp. 88-109, 2012. (in Russian).

[6] S. V. Boyko, V. V. Mischenko, G. V. Sandrakov, "The numerical investigation method for evaporated plasma," Journal Num. and Appl. Math., no. 2 (95) , pp. 3-12, 2007.

[7] G. V. Sandrakov, Modeling of Hydrodynamics Processes with Phase Transition, "Information Technologies and Computer Modelling" Proc Int. Scient. Conference, Ivano-Frankivsk, 14-19 May, pp. 303-306, 2018.

[8] G. R. Liu, M. B. Liu, Smoothed particle hydrodynamics. A meshfree particle method.World Scientic Publishing, New Jersey, 2003. 